\title{
Parasitologic Assessment of Two-Dose and Monthly Intermittent Preventive Treatment of Malaria during Pregnancy with Sulphadoxine-Pyrimethamine (IPTP-SP) in Lagos, Nigeria
}

\author{
Chimere O. Agomo, ${ }^{1,2}$ Wellington A. Oyibo, ${ }^{1}$ and Funke Odukoya-Maije ${ }^{3}$ \\ ${ }^{1}$ Tropical Diseases Laboratory, Department of Medical Microbiology and Parasitology, College of Medicine, University of Lagos, \\ Lagos, Nigeria \\ ${ }^{2}$ Malaria Research Laboratory, Department of Biochemistry, Nigerian Institute of Medical Research, P.M.B 2013 Yaba, Lagos, Nigeria \\ ${ }^{3}$ Department of Obstetrics and Gynaecology, Ajeromi General Hospital, Ajegunle, Lagos, Nigeria
}

Correspondence should be addressed to Wellington A. Oyibo, waoyibo@gmail.com

Received 25 May 2011; Revised 10 August 2011; Accepted 30 August 2011

Academic Editor: Mats Wahlgren

Copyright ( $\odot 2011$ Chimere O. Agomo et al. This is an open access article distributed under the Creative Commons Attribution License, which permits unrestricted use, distribution, and reproduction in any medium, provided the original work is properly cited.

Intermittent preventive treatment of malaria with sulphadoxine-pyrimethamine (IPTP-SP) is a key strategy in the control of malaria in pregnancy. However, reports of increasing level of resistance to SP using nonpregnant populations have made it imperative for the continuous monitoring of the efficacy of SP in pregnant women. This study assessed using microscopy, monthly dosing and the standard two-dose regimen among 259 pregnant women attending antenatal clinics in Lagos, Nigeria that consented 122 in the two-dose arm (Arm A) and 137 in the monthly dose arm (Arm B). Baseline parasitaemia in the two groups was 5 (4.1\%) and $3(2.2 \%)$ in Arms A and B, respectively. Few of the women developed parasitaemia after the initial SP dose in Arms A 4 (3.3\%) and B 2 (1.5\%). However, none of the women had malaria infection after the second dose in both Arms. Although IPTP-SP is suggestive of protecting the women from malaria infection, there was no significant difference observed between the two dosing schemes.

\section{Introduction}

An estimated 25-30 million women become pregnant annually in malaria-endemic areas of Africa, most of them living in areas of stable malaria transmission [1]. The immunosuppression associated with pregnancy and the absence of specific immunity to the unique subset of parasites (VAR2CSA) that sequester in the placenta, especially in primigravidae, are the reasons for the increased susceptibility of pregnant women to malaria infection $[2,3]$. However, the antidisease immunity acquired prior to pregnancy keeps the infection asymptomatic in presentation. However, the subclinical infection still poses a great danger to both the mother and the foetus. The presence of parasites in the placenta can lead to maternal anaemia (potentially responsible for maternal death when severe), low birth weight (LBW), congenital malaria, premature delivery, abortion, and stillbirth [4-6].
Current strategies to control malaria in pregnancy are the intermittent preventive treatment with sulphadoxinepyrimethamine (IPTp-SP), use of insecticide-treated bed nets, and case management of malaria illness and anemia [ 1 , 7]. IPTp-SP is the administration of two or more therapeutic doses of SP regardless of the presence of malarial infection, at an interval of at least four weeks, starting in the second trimester of pregnancy (after quickening). The first SP dose is administered in the second trimester after quickening and the second dose of SP in the third trimester to ensure that the placenta is cleared of malaria parasites at the time of rapid foetal growth [8]. Two doses of SP are recommended for HIV-negative pregnant women while HIV-positive women are to receive 3 doses of SP [7].

Prior to the introduction of IPTp-SP, antimalaria chemoprophylaxis during pregnancy was based on administration of weekly pyrimethamine or chloroquine (CQ) [9]. The 
effectiveness of weekly chemoprophylaxis with CQ and pyrimethamine was marred by poor compliance and emergence of $P$. falciparum strains that are resistant to these drugs $[10,11]$. Apart from lower cost and higher compliance, IPTpSP is significantly better than CQ at reducing the rate of placental infection, anaemia, and low birth weight [12-14]. Weekly prophylaxis with SP is not recommended because of the high likelihood of fatal reactions such as Steven-Johnson's syndrome resulting from frequent administration of SP [15].

Sulphadoxine-pyrimethamine is an antifolate drug. Folic acid is needed by the malaria parasites for the biosynthesis of purines and pyrimidines needed for DNA synthesis and cell multiplication. The combination of pyrimethamine and sulphadoxine thus offers a two-step synergistic inhibition of the bifunctional enzyme dihydrofolate reductase-thymidylate synthase (DHFR-TS) and dihydropteroate synthase (DHPS) in the folate synthetic pathway. This leads to a failure of nuclear division and subsequent cell death $[16,17]$.

The efficacy of IPTpSP results from intermittent clearance of parasitaemia and mostly through posttreatment prophylaxis, provided that effective antiparasitic concentrations of the drug were maintained during the entire dosing interval [8]. Sulphadoxine-pyrimethamine is well absorbed after oral administration, achieving peak plasma levels within 2 to $8 \mathrm{~h}$, have $>90 \%$ bioavailability, and are $85-90 \%$ protein bound [18]. Sulphadoxine is metabolized via glucuronidation and excreted primarily in urine. Pyrimethamine is metabolized to several metabolic products, and $\sim 15-30 \%$ is excreted unchanged in urine [19]. The mean terminal elimination half-lives are 200 and $100 \mathrm{~h}$ for sulphadoxine and pyrimethamine, respectively, in healthy nonpregnant adults $[20,21]$.

IPTp-SP efficacy depends on the maintenance of antiparasitic concentrations over the dosing intervals. SP has a good safety profile after the first trimester, has not been associated with adverse effects on the foetus, and is well tolerated by mothers. The estimated risk of severe adverse reactions to IPTp-SP (monthly or less often) is 0-6.3\% [22]. Fatal adverse reactions to SP were estimated to be 0.11 deaths $/ 100,000$ SP exposures $[23,24]$. The administration of SP in the first trimester is not advised because of potential teratogenic effect on the foetus [7].

Some reports suggest that monthly IPTp-SP may be more appropriate in areas that are hyper- and holoendemic for malaria $[25,26]$. Parasitological failure rate of $24.4 \%$ on day 14 of SP has been reported among children aged 6 months to five years with acute uncomplicated malaria in Southwest Nigeria [27]. It is therefore important to determine the parasitologic outcome with the 2-dose regimen in protecting pregnant women from malaria in Lagos (a holoendemic area), especially in the context where data on parasitologic monitoring of IPTp-SP in Nigeria is unavailable. A malaria prevalence rate of $7.7 \%$ among pregnant women attending these health facilities had been reported previously [28].

This paper reports the outcome of parasitologic assessment of the standard 2-dose regimen and the monthly dose of IPTp-SP in preventing malaria parasitaemia during pregnancy in Lagos, Nigeria, in the light of documented SP resistance reports in children and the adult population.

\section{Materials and Methods}

The field study was conducted in Lagos, Nigeria, specifically at the Ajeromi General Hospital, Ajegunle, a government secondary health facility and the St. Kizito Primary Healthcare Center, Lekki, a non-for-profit primary health facility. Routine antenatal care in these facilities included: monitoring of body weight, blood pressure, haemoglobin level, and urinalysis. Health talks were given at each clinic day. The women received haematinics Folic acid tablets as routine care in pregnancy. HIV counseling and testing along with screening for sexually transmitted diseases are part of routine preconditions for receiving antenatal services in these hospitals. The laboratory studies were done at The International Malaria Microscopy Training Centre, Tropical Diseases Research Laboratory, Department of Medical Microbiology and Parasitology, College of Medicine of the University of Lagos, Idi-Araba, Lagos, Nigeria.

The population of pregnant women in Lagos is approximately 640,000 (i.e., $4 \%$ of the total population). Assuming a worst cure rate of $50 \%$ for SP at $95 \%$ confidence interval, precision of $10 \%$, and a dropout rate of $10 \%$, a minimum sample size of 60 is needed for each arm giving a total of 120 pregnant women.

2.1. Enrolment of Study Participants. Pregnant women attending the antenatal clinics (ANCs) who consented to participate in the study were recruited at booking (first registration, usually after 16 weeks). The study participants were given study numbers and personal identifiers removed to ensure confidentiality. Access to study records was restricted. The inclusion criteria were (a) pregnancy at second trimester (b) no history of allergic reaction to sulphonamides, and (c) HIV negative. The exclusion criteria were (a) positive test for VDRL, diabetics, and hypertension (essential or pregnancyinduced) (b) severe anaemia (PCV $<20 \%$ ) (c) multiple pregnancy as determined by an ultrasound scan report and other severe illness that could require hospitalization. The women were given a dose of SP (1500 mg sulphadoxine and $75 \mathrm{mg}$ pyrimethamine) in three tablets. The SP was obtained directly from the manufacturer.

The pregnant women were randomly assigned into two major arms: Arm A received the standard 2 doses of SP and Arm B received monthly dose of SP. The randomization was done by asking the women to pick up shuffled envelopes containing the study arms. In addition, the laboratory staffs including the microscopists were blinded to the allocations of the patients. In Arm A, the study participants were given a treatment dose of SP (3 tablets each containing $500 \mathrm{mg}$ sulphadoxine and $25 \mathrm{mg}$ pyrimethamine) in the second trimester and another treatment dose in the third trimester. In Arm B, the participants received a treatment dose of SP monthly. In both arms, parasitaemia were assessed monthly. All the participants were parasitologically and clinically monitored at each visit before delivery.

The indicators used in this study were parasite clearance, monitoring of parasitaemia, and delivery outcome. The parasite clearance was assessed by the proportion of women that were parasitaemic who became aparasitaemic after the 
intake of SP. Monitoring of parasitaemia was done monthly before SP administration. The delivery outcome was assessed by the number of live births and low birth weight babies. Low birth weight was defined as birth weight less than $2.5 \mathrm{~kg}$. In addition, demographic and other information including insecticide-treated bed nets' use were collected through a questionnaire that was administered.

2.2. Collection of Samples. Venous blood samples $(\sim 2 \mathrm{~mL})$ were collected from the participants at recruitment and at each antenatal visit from their 2 nd trimester. Two slides of thin and thick films (on the same slide) were made for each study participant.

2.3. Followup of Study Participants. All the study participants were monitored monthly for malaria parasitaemia. Those that missed appointment days were contacted by phone and appointments rescheduled within one week. Alternatively, they were visited at home. The administration of SP was by directly observed treatment (DOT) method. The SP was directly obtained from the manufacturers. At each monthly visit, routine clinical assessments for safety were conducted on the pregnant women and blood collected for parasitologic and other investigations. The month of recruitment was termed Month 0 (M0). Subsequent follow-up months were termed M1, M2, M3, and M4. The participant that became positive with malaria parasite during followup was treated with oral quinine (600 mg, three times daily for one week).

At delivery, placental blood samples were obtained by making an incision on the placenta tissue (maternal surface) and blood collected with a $2 \mathrm{~mL}$ syringe. Infants were weighed with a digital scale at birth.

2.4. Laboratory Methods. Thin and thick smears were prepared for each participant following standard procedures for malaria microscopy. Two competent microscopists read each slide, and when there was discordance in reading, a third microscopist reread the slide and served as a tie breaker. Essentially, the discordance level for the acceptance of any two parasite counts was set at less than $20 \%$. The packed cell volume (PCV) was determined using standard protocol. Anaemia in pregnancy was defined as PCV $<33 \%$ [29].

2.5. Ethical Consideration. Approvals to conduct this study were obtained from the Research Grants and Experimentation Ethics Committee, College of Medicine of the University of Lagos and the Institutional Review Board of the Nigerian Institute of Medical Research, Lagos. Generally, the protocol was conducted in accordance with Good Clinical Practice (GCP), Good Clinical Laboratory Practices (GCLPs) and indeed the tenets of the Helsinki Declaration of 1964 as amended in 2000.

2.6. Data Analysis. The data generated from the study were analyzed using EPIINFO 2002 statistical software (CDC, Atlanta, GA, USA). Tests for associations and differences were done by chi-square analysis, Fischer Exact test, and analysis of variance where done as appropriate. $P$ value less than 0.05 was taken as significant.

\section{Results}

A total of 259 pregnant women who were in their second trimester were enrolled: $122(47.1 \%)$ in Arm A (standard 2dose IPTp-SP) and 137 (52.9\%) in Arm B (monthly IPTpSP). The study participants in the two arms of the study had similar baseline characteristics (Table 1) $(P>0.05)$.

The number of pregnant women with parasitaemia at enrolment (M0) was $5(4.1 \%)$ and $3(2.2 \%)$ in Arms A and $\mathrm{B}$, respectively. The mean parasitaemia at M0 was 777.4 parasites $/ \mu \mathrm{L}$ (range, $111-2,063$ parasites $/ \mu \mathrm{L}$ ) and 691.7 parasites $/ \mu \mathrm{L}$ (range, $127-1,664$ ) in Arm A and B, respectively, but reduced at M1 in both Arms to298.3 parasites/ ${ }^{-} \mathrm{L}$ (range, 149-390 parasites $/ \mu \mathrm{L}$ ) and cleared at M2 in Arm B. Few of the women developed parasitaemia after the initial SP dose in Arms A, $4(3.3 \%)$ and B, $2(1.5 \%)(P=0.577)$. None of the women in both Arms of the study developed parasitaemia after the second dose of SP (which for Arm A was from M2). The proportion of women infected was reduced to $20 \%$ and $0 \%$ in Arms A and B, respectively, (Figure 1). None of the study participant developed clinical malaria during the followup.

In a total of 12 placental blood samples collected from women in Arm B. none (0.0\%) of the samples had malaria parasitaemia.

The result of pregnancy outcome was obtained from a subset of 156 of the 259 women studied. The birth outcome was assessed based on the proportion of live birth and weight of the babies. There were a total of 149 (95.5\%) live births and 7 (4.5\%) deaths recorded. Of the foetal deaths, $3(2.9 \%)$ were from women in monthly IPTp-SP and 4 (7.5\%) from women in the 2-Dose IPTp-SP. There was no statistical association $(P=0.178)$ between the proportion of deaths and the study arms. The mean weight of the babies with LBW was $1.9 \pm 0.1 \mathrm{~kg}$ and $2.0 \pm 0.4 \mathrm{~kg}$ (range 1.4-2.3 kg) in Arms A and $B$ respectively while the babies with normal birth weight (NBW) had a mean weight of $3.3 \pm 0.4 \mathrm{~kg}$ and $3.3 \pm 0.4 \mathrm{~kg}$ in Arms A, and B, respectively. The proportion of LBW in the monthly IPTp-SP (Arm B) (5 (5.0\%)) was similar to the standard 2-dose IPTp-SP (Arm A) $(2(4.1 \%))(P=0.581)$.

The age and gravidity of women whose babies died were not significantly associated $(P=0.907)$ with the study arms. Also, the age and gravidity of women who had LBW babies were not significantly associated with the IPTp-SP dosing schemes.

\section{Discussion}

The administration of IPTp-SP was effective in protecting $4(3.3 \%)$ in Arm A and 2 (1.5\%) in Arm B of the study participants from malaria infection and clinical malaria. A similar finding was reported by Nyunt and colleagues [30] where 1 of the 98 pregnant women who were on IPTpSP became parasitemic, none developed clinical malaria during the period of followup and also none of the patients tested positive for $P$. falciparum on assessment of placental 
TABLE 1: Baseline characteristics of the study participants.

\begin{tabular}{lcc}
\hline & Arm A & Arm B \\
\hline Number & 122 & 137 \\
Mean age (years) & $26.5 \pm 4.2$ & $27.9 \pm 4.9$ \\
Gravidity & & \\
$\quad$ Primigravidae & $47(38.6 \%)$ & $56(40.9 \%)$ \\
Secundigravidae & $33(27.0 \%)$ & $35(25.5 \%)$ \\
Multigravidae & $42(34.4 \%)$ & $46(33.6 \%)$ \\
Mean PCV(\%) & $32.3 \pm 3.7 \%$ & $32.4 \pm 3.7 \%$ \\
PCV $<33 \%$ & $29(23.8 \%)$ & $29(21.2 \%)$ \\
PCV $\geq 33 \%$ & $93(76.2 \%)$ & $108(78.8 \%)$ \\
\hline
\end{tabular}

Arm A: standard 2-Dose IPTp-SP; Arm B: monthly IPTp-SP.

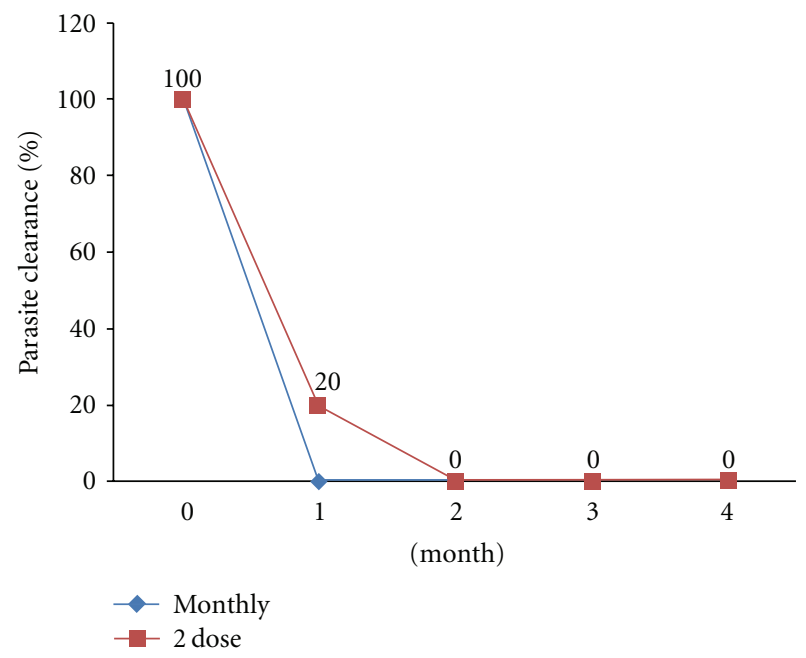

FIgUre 1: Parasite clearance in the monthly and 2-dose IPTp-SP groups. Fisher Exact $P=0.625$.

parasitaemia. This is suggestive of the effectiveness of SP in preventing malaria infection among pregnant women in Lagos despite reports of high parasitological failure rate of SP, $24.4 \%$ reported in children in southwest Nigeria [27]. The difference in response to treatment with SP in children and pregnant women can be explained by the report that parasitological response is significantly better in pregnant women than in children treated with same drug [31]. The reasons for the high efficacy in pregnant women can be attributed to the presence of acquired immunity which assists the drug in clearing malaria parasites as well as providing a prophylactic effect $[27,32]$. Kalanda et al. [33] reported that pregnant women (all gravidae) responded better than children to treatment with chloroquine and sulphadoxine-pyrimethamine. Thus, the greater drug sensitivity in pregnant women probably indicates differences in host susceptibility rather than parasite resistance. The absence of parasitaemia after M1 in monthly IPTp-SP and M2 in the standard 2-dose IPTp-SP agrees with the findings of other investigators that the administration of at least two therapeutic doses of IPTp-SP after the first trimester is effective in preventing maternal and placental malaria as well as improving pregnancy outcomes among parturient women as reported in Kenya [34, 35].

The low parasitaemia and the clearance observed during follow-up months among women who were parasitaemic at M0, and in women who became parasitaemic after the first dose of SP, suggest that SP, in addition to other factors such as existing maternal immunity could have provided a suppressive effect on parasite multiplication.

The proportion of women who became parasitaemic during the follow-up visits for monthly monitoring of parasitaemia $2(1.5 \%)$ and $3(2.5 \%)$ in the monthly and the standard 2-dose IPTp-SP groups, respectively, were similar at Month $1(P=0.445)$. The only pregnant woman that had parasitaemia at Month 2 did not receive SP on Month 1. Nevertheless, on administration of a therapeutic dose of $\mathrm{SP}$, this parasitaemia cleared at the next month's visit. This finding may suggest that the level of SP in the blood was not able to prevent the multiplication of the parasites. In the presence of drug resistance, the drug level needed to either suppress or prevent infection is increased [32]. Of the women studied, only $11 \%$ of them slept under insecticide treated net.

Based on parasitaemia, the monthly dosing was not significantly superior than the 2-dose regimen $(P>0.05)$. The low number of infected patients in the study may not, however, permit a more conclusive statement. However, similar finding by Hamer and colleagues [26] indicated that the monthly dosing was not more efficacious than the standard 2 -dose regimen in an area where malaria transmission is mesoendemic. Contrarily, Filler and coworkers [25] reported that monthly IPTp-SP was a better option than 2-dose IPTp$\mathrm{SP}$ in areas hyperendemic for malaria. Therefore, considering the $7.7 \%$ prevalence of malaria in pregnancy in this study, Lagos currently appears to be mesoendemic for malaria.

The outcome of the pregnancy was assessed based on two criteria: (a) if the pregnancy resulted in the birth of a live baby or not and (b) the birth weight of the live baby. In this study, though the proportion of deaths was higher in the 2-dose IPTp-SP than in the monthly IPTp-SP, 3, the difference was not statistically significant and was not likely to have been differentially influenced by any of the dosing regimens. The effect of the different dosing regimens on low birth weight was similar $(P=0.581)$ and consistent with the reports in Zambia by Gill and co-workers [36] and Hamer and colleagues [26].

Of the 12 women assessed for placental parasitaemia in the monthly IPTp-SP arm, none (0) had malaria parasites. However, the unwillingness of the women to permit placental biopsies (though they agreed at enrolment) and their deliveries at other health facilities limits the interpretation of the data obtained from the placental samples in this study.

In conclusion, Sulphadoxine-pyrimethamine could be said to be effective in protecting pregnant women against malaria infection in Lagos using the limited data available in the light of heightened fears of resistance and threatened efficacy when used as IPTp. Monthly IPTp-SP could be said to be equivalent to the standard 2-dose regimen in protecting pregnant women against malaria infection and in the birth outcome. 


\section{Conflict of Interests}

The authors declare that they have no conflict of interests.

\section{Authors' Contributions}

C. O. Agomo and W. A. Oyibo conceptualized and designed the study. F. Odukoya-Maije clinically assessed the patients. C. O. Agomo and W. A. Oyibo conducted the monthly monitoring of malaria parasitaemia and other laboratory investigations. C. O. Agomo analyzed the data generated. C. Agomo, W. A. Oyibo and F. Odukoya-Meije prepared and proof-read the manuscript.

\section{Acknowledgment}

The authors thank the management and staff of St. Kizito Primary Health Centre, Lekki and the Ajeromi General Hospital, Ajegunle, Lagos. Particularly, they appreciate the contributions of the Matrons and midwives at the antenatal clinics in these hospitals. they thank Emzor Pharmaceuticals, Lagos and IPCA Pharmaceuticals, Lagos for providing the sulphadoxine-pyrimethamine (SP) that was used for the study. The contributions of Mr. Raji Onimisi and Mr. Essien Akpan in following up the pregnant women are acknowledged.

\section{References}

[1] World Health Organization, "A strategic framework for malaria prevention and control during pregnancy in the African region," Tech. Rep. AFR/MAL/04/01, WHO Regional Office for Africa, Brazzaville, Republic of Congo, 2004.

[2] M. Fried and P. E. Duffy, "Adherence of Plasmodium falciparum to chondroitin sulfate A in the human placenta," Science, vol. 272, no. 5267, pp. 1502-1504, 1996.

[3] L. Hviid and A. Salanti, "VAR2CSA and protective immunity against pregnancy-associated Plasmodium falciparum malaria," Parasitology, vol. 134, no. 13, pp. 1871-1876, 2007.

[4] R. Steketee, J. Wirima, A. Hightower, L. Slutsker, D. L. Heymann, and J. G. Breman, "The effect of malaria and malaria prevention in pregnancy on offspring birthweight, prematurity, and intrauterine growth retardation in Rural Malawi," American Journal of Tropical Medicine and Hygiene, vol. 55, no. 1, pp. 33-41, 1996.

[5] R. D. Newman, A. Hailemariam, D. Jimma et al., "Burden of malaria during pregnancy in areas of stable and unstable transmission in Ethiopia during a nonepidemic year," Journal of Infectious Diseases, vol. 187, no. 11, pp. 1765-1772, 2003.

[6] S. J. Rogerson and P. Boeuf, "New approaches to pathogenesis of malaria in pregnancy," Parasitology, vol. 134, no. 13, pp. 1883-1893, 2007.

[7] Federal Ministry of Health, "National guidelines and strategies for malaria prevention and control during pregnancy," Tech. Rep., Federal Ministry of Health, Nigeria, 2005.

[8] N. J. White, "Intermittent presumptive treatment for malaria: a better understanding of the pharmacodynamics will guide more rational policymaking," PLoS Medicine, vol. 2, pp. 2833, 2005.
[9] World Health Organization, "WHO expert committee on malaria. Eighteenth report," WHO Technical Report Series, World Health Organization, Geneva, Switzerland, 1986.

[10] B. L. Nahlen, T. Alakija, O. Ogunbode et al., "Lack of efficacy of pyrimethamine prophylaxis in pregnant Nigerian women," The Lancet, vol. 2, no. 8667, pp. 830-834, 1989.

[11] S. B. Sirima, R. Sawadogo, A. C. Moran et al., "Failure of a chloroquine chemoprophylaxis program to adequately prevent malaria during pregnancy in Koupela district, Burkina Faso," Clinical Infectious Diseases, vol. 36, no. 11, pp. 13741382, 2003.

[12] M. Diallo, C. A. Dabo, R. Saye et al., "Randomized clinical trial of two malaria prophylaxis regimens for pregnant women in Faladie, Mali," Medecine Tropicale, vol. 67, no. 5, pp. 477-480, 2007.

[13] K. Kayentao, M. Mungai, M. Parise et al., "Assessing malaria burden during pregnancy in Mali," Acta Tropica, vol. 102, no. 2, pp. 106-112, 2007.

[14] I. U. Tukur, T. D. Thacher, A. S. Sagay, and J. K. Madaki, "A comparison of sulfadoxine-pyrimethamine with chloroquine and pyrimethamine for prevention of malaria in pregnant Nigerian women," American Journal of Tropical Medicine and Hygiene, vol. 76, no. 6, pp. 1019-1023, 2007.

[15] H. O. Alkadi, "Antimalarial drug toxicity: a review," Chemotherapy, vol. 53, no. 6, pp. 385-391, 2007.

[16] D. J. Bzik, W. B. Li, T. Horii, and J. Inselburg, "Molecular cloning and sequence analysis of the Plasmodium falciparum dihydrofolate reductase-thymidylate synthase gene," Proceedings of the National Academy of Sciences of the United States of America, vol. 84, no. 23, pp. 8360-8364, 1987.

[17] C. H. Sibley, J. E. Hyde, P. F. Sims et al., "Pyrimethaminesulfadoxine resistance in Plasmodium falciparum: what next?" Trends in Parasitology, vol. 17, no. 12, pp. 582-588, 2001.

[18] C. R. Jones and S. M. Ovenell, "Determination of plasma concentrations of dapsone, monoacetyl dapsone and pyrimethamine in human subjects dosed with maloprim," Journal of Chromatography, vol. 163, no. 2, pp. 179-185, 1979.

[19] M. D. Coleman, G. W. Mihaly, G. Edwards, R. E. Howells, and A. M. Breckenridge, "The disposition of pyrimethamine base and pyrimethamine pamoate in the mouse: effect of route of administration," Biopharmaceutics and Drug Disposition, vol. 7, no. 2, pp. 173-182, 1986.

[20] M. D. Edstein, "Pharmacokinetics of sulfadoxine and pyrimethamine after Fansidar administration in man," Chemotherapy, vol. 33, no. 4, pp. 229-233, 1987.

[21] E. Weidekamm, H. Plozza-Nottebrock, I. Forgo, and U. C. Dubach, "Plasma concentrations of pyrimethamine and sulfadoxine and evaluation of pharmacokinetic data by computerized curve fitting," Bulletin of the World Health Organization, vol. 60, no. 1, pp. 115-122, 1982.

[22] P. E. Brentlinger, C. B. Behrens, and M. A. Micek, "Challenges in the concurrent management of malaria and HIV in pregnancy in sub-Saharan Africa," Lancet Infectious Diseases, vol. 6, no. 2, pp. 100-111, 2006.

[23] F. Forna, M. McConnell, F. N. Kitabire et al., "Systematic review of the safety of trimethoprim-sulfamethoxazole for prophylaxis in HIV-infected pregnant women: implications for resource-limited settings," AIDS Reviews, vol. 8, no. 1, pp. 24-36, 2006.

[24] J. E. Gimnig, J. R. MacArthur, M. M'bang'ombe et al., "Severe cutaneous reactions to sulfadoxine-pyrimethamine and trimethoprim-sulfamethoxazole in Blantyre District, Malawi," American Journal of Tropical Medicine and Hygiene, vol. 74, no. 5, pp. 738-743, 2006. 
[25] S. J. Filler, P. Kazembe, M. Thigpen et al., "Randomized trial of 2-dose versus monthly sulfadoxine-pyrimethamine intermittent preventive treatment for malaria in HIV-positive and HIV-negative pregnant women in Malawi," Journal of Infectious Diseases, vol. 194, no. 3, pp. 286-293, 2006.

[26] D. H. Hamer, V. Mwanakasale, W. B. MacLeod et al., "Twodose versus monthly intermittent preventive treatment of malaria with sulfadoxine-pyrimethamine in HIV-seropositive pregnant Zambian women," Journal of Infectious Diseases, vol. 196, no. 11, pp. 1585-1594, 2007.

[27] Federal Ministry of Health, "National antimalarial treatment policy," Federal Ministry of Health Nigeria, National Malaria and Vector Control Division Abuja, Nigeria, 2005.

[28] C. O. Agomo, W. A. Oyibo, R. I. Anorlu, and P. U. Agomo, "Prevalence of malaria in pregnant women in Lagos, SouthWest Nigeria," Korean Journal of Parasitology, vol. 47, no. 2, pp. 179-183, 2009.

[29] World Health Organization, Preventing and Controlling Iron Deficiency Anaemia Through Primary Health Care, WHO publications, Geneva, Switzerland, 1989.

[30] M. M. Nyunt, I. Adam, K. Kayentao et al., "Pharmacokinetics of sulphadoxine and pyrimethamine in intermittent preventive treatment of malaria in pregnancy," Clinical Pharmacology and Therapeutics, vol. 87, no. 2, pp. 226-234, 2010.

[31] T. K. Mutabingwa, K. Muze, R. Ord et al., "Randomized trial of artesunate+amodiaquine, sulfadoxine-pyrimethamine+amodiaquine, chlorproguanal-dapsone and SP for malaria in pregnancy in Tanzania," PLoS ONE, vol. 4, no. 4, Article ID e5138, 2009.

[32] P. Bloland, "Drug resistance in malaria," WHO Monograph WHO/CDS/CSR/DRS/2001.4, World Health Organization, Geneva, Switzerland, 2001.

[33] G. Kalanda, J. Hill, F. Verhoeff, and B. Brabin, "Comparative efficacy of chloroquine and sulphadoxine-pyrimethamine in pregnant women and children: a meta-analysis," Tropical Medicine and International Health, vol. 11, no. 5, pp. 569-577, 2006.

[34] M. E. Parise, J. G. Ayisi, B. L. Nahlen et al., "Efficacy of sulfadoxine-pyrimethamine for prevention of placental malaria in an area of Kenya with a high prevalence of malaria and human immunodeficiency virus infection," American Journal of Tropical Medicine and Hygiene, vol. 59, no. 5, pp. 813-822, 1998.

[35] A. M. van Eijk, J. G. Ayisi, F. O. ter Kuile et al., "Effectiveness of intermittent preventive treatment with sulphadoxinepyrimethamine for control of malaria in pregnancy in western Kenya: a hospital-based study," Tropical Medicine and International Health, vol. 9, no. 3, pp. 351-360, 2004.

[36] C. J. Gill, W. B. MacLeod, V. Mwanakasale et al., "Inferiority of single-dose sulfadoxine-pyrimethamine intermittent preventive therapy for malaria during pregnancy among HIVpositive Zambian women," Journal of Infectious Diseases, vol. 196, no. 11, pp. 1577-1584, 2007. 


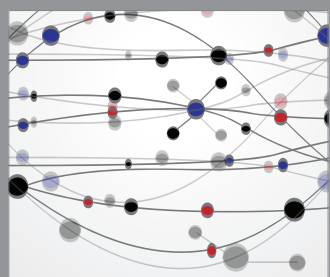

The Scientific World Journal
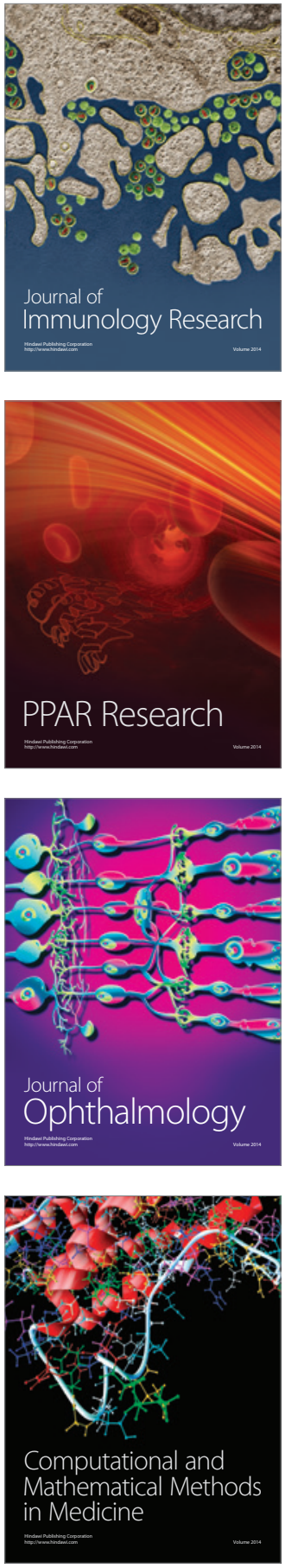

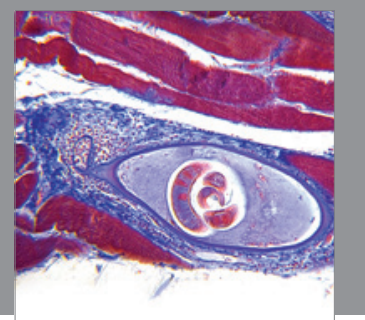

Gastroenterology

Research and Practice
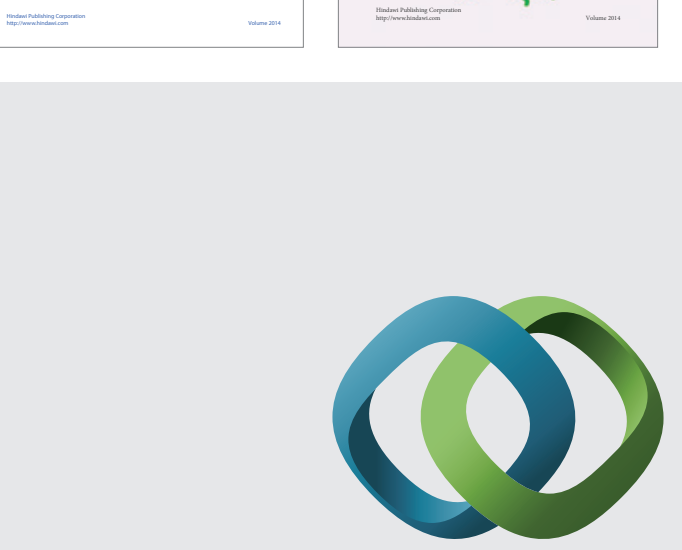

\section{Hindawi}

Submit your manuscripts at

http://www.hindawi.com
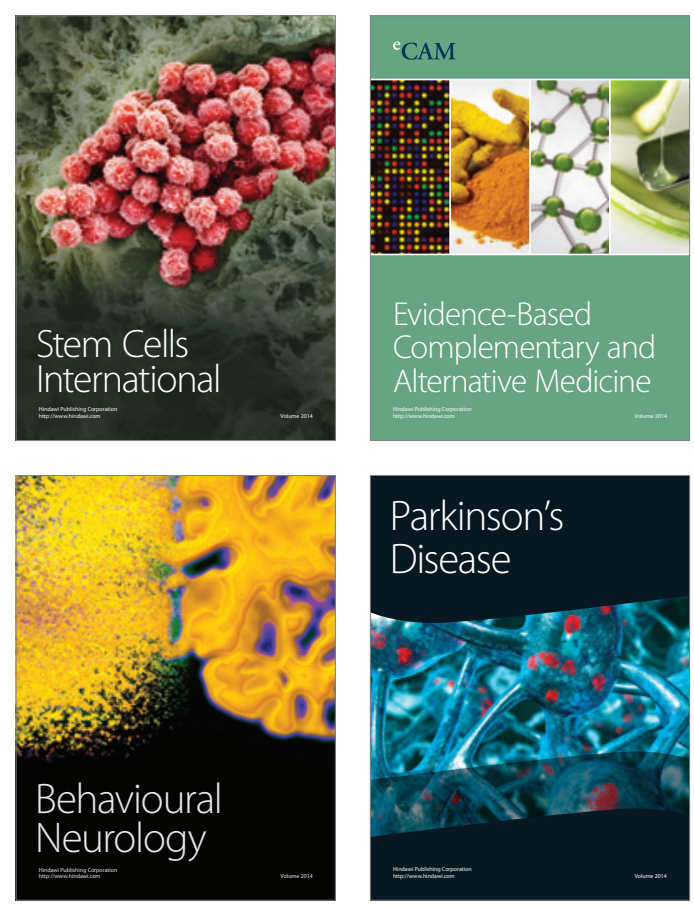

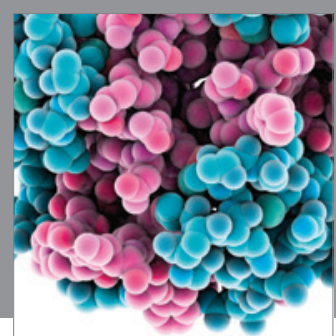

Journal of
Diabetes Research

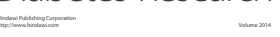

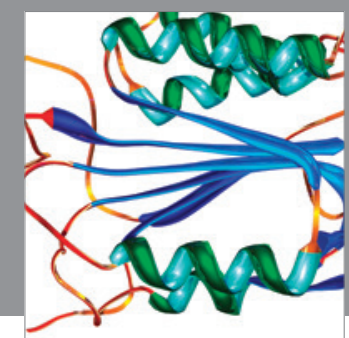

Disease Markers
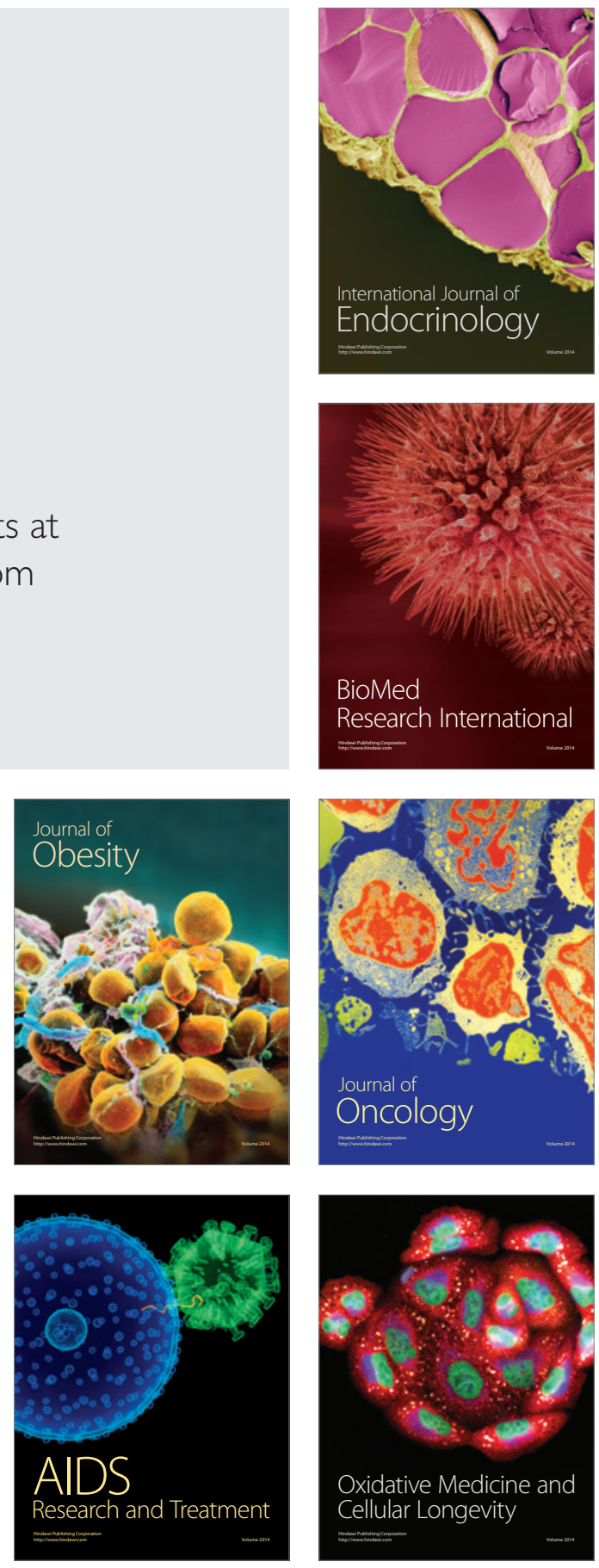